\title{
Body Mass Index Best Predicts Recovery of Recombinant Factor VIII in Underweight to Obese Patients with Severe Haemophilia A
}

\author{
Andreas Tiede ${ }^{1}$ Ana Rosa Cid ${ }^{2}$ Georg Goldmann ${ }^{3}$ \\ Toshko Lissitchkov ${ }^{6}$ Marcus May ${ }^{7}$ Irina Matytsina ${ }^{8}$ \\ ${ }^{1}$ Hematology, Hemostasis, Oncology and Stem Cell Transplantation \\ Unit, Hannover Medical School, Hannover, Germany \\ 2 Thrombosis and Haemostasis Unit, Hospital Universitario y \\ Politécnico La Fe, Valencia, Spain \\ 3 Institute of Experimental Haematology and Transfusion Medicine, \\ University of Bonn, Bonn, Germany \\ ${ }^{4}$ Hospital Universitario La Paz, Autónoma University, Madrid, Spain \\ ${ }^{5}$ Quanticate Ltd, Hitchin, United Kingdom \\ ${ }^{6}$ Clinic of Haematology, Specialized Hospital for Active Treatment of \\ Haematological Diseases, Sofia, Bulgaria \\ ${ }^{7}$ Clinical Research Center Hannover, Hannover Medical School, \\ Hannover, Germany \\ 8 Novo Nordisk A/S, Søborg, Denmark \\ ${ }^{9}$ Clinic of Haematology, Faculty of Medicine, University of Belgrade, \\ Belgrade, Serbia \\ ${ }^{10}$ Clinical Division of Haematology and Haemostaseology, Medical \\ University of Vienna, Vienna, Austria
}

Thromb Haemost 2020;120:277-288.
Victor Jiménez-Yuste ${ }^{4}$ Michael Pluta ${ }^{5}$

Predrag Miljic ${ }^{9}$ Ingrid Pabinger ${ }^{10}$ Paula Persson ${ }^{8}$

Address for correspondence Andreas Tiede, MD, PhD, Hematology, Hemostasis, Oncology and Stem Cell Transplantation Unit, Hannover Medical School, Carl-Neuberg-Street 1, 30625 Hannover, Germany (e-mail: Tiede.Andreas@mh-hannover.de).

\begin{abstract}
Keywords

- body mass index

- dosing model

- recombinant factor VIII

- haemophilia

- pharmacokinetics
\end{abstract}

Background Factor VIII (FVIII) products are usually dosed according to body weight (BW). This may lead to under- or over-dosing in underweight or obese patients, respectively.

Objective This article evaluates the pharmacokinetics (PK) of recombinant FVIII concentrate, particularly recovery, in relation to body mass index (BMI) and other body composition descriptors.

Materials and Methods Thirty-five previously treated adults with severe haemophilia A from five BMI categories (underweight, normal, overweight, obese class I and II/III) were included. PK was evaluated after $50 \mathrm{IU}$ per kilogram of BW single-dose recombinant FVIII (turoctocog alfa). The body composition variable was based on measurements of weight, height, bioimpedance analysis, and dual-energy X-ray absorptiometry. A dosing model was derived to achieve similar peak FVIII activity levels across BMI categories.

Results A statistically significant positive association between $B M I$ and $C_{30 m i n}$, $\mathbb{R}_{30 \mathrm{~min}}$, and $A \cup C_{0-i n f}$ was observed; $C L$ and $V_{s s}$ showed a significant negative association with $\mathrm{BMI}$; $\mathrm{t}_{1 / 2}$ was independent of $\mathrm{BMI}$ and other parameters. The dosing model introduced a correction factor ' $M$ ' for each BMI category, based on linear regression analysis of $C_{30 \text { min }}$ against $B M I$, which ranged from 0.55 for underweight to 0.39 for received

May 13, 2019

accepted after revision October 19, 2019
DOI https://doi.org/

$10.1055 / \mathrm{s}-0039-3400745$.

ISSN $0340-6245$.
(C) 2020 Georg Thieme Verlag KG Stuttgart · New York
License terms

(c) $(\oplus \circledast \$$ 
obese class II/III. This model achieved similar peak FVIII activity levels across BMI categories, estimating an average dose adjustment of $+243.3 \mathrm{IU}$ (underweight) to $-1,489.6$ IU (obese class II/III) to achieve similar $C_{30 \mathrm{~min}}$.

Conclusion BMI appears to be the best predictor of recombinant FVIII recovery; however, PK endpoints were also dependent on other body composition variables. The model demonstrated that dosing can be adjusted for individual BMI to achieve better FVIII predictability across BMI categories.

\section{Introduction}

Factor VIII (FVIII) products have a long-standing and wellestablished role in the treatment of haemophilia $A$ as long-term prophylaxis to protect against bleeding episodes, for on-demand treatment to control a bleed or for providing haemostatic cover for surgery. ${ }^{1}$ Correct dosing of FVIII is crucial to avoid under-dosing, which may lead to inadequate bleed control, or overdosing leading to a waste of FVIII product. ${ }^{2}$

FVIII products are typically dosed according to total body weight $(\mathrm{BW}){ }^{2,3}$ meaning that heavier patients receive a proportionally higher drug dose than patients with normal weight. ${ }^{4}$ The growing population of overweight and obese patients with haemophilia is a clinical concern, ${ }^{3,5}$ and arthropathy occurs more commonly in overweight and obese patients with haemophilia; ${ }^{6}$ though it is unclear why joint disease is increased in this patient population. ${ }^{4}$

FVIII is primarily distributed in plasma, with only a small fraction ( $\sim 14 \%$ ) circulating outside the vascular system. ${ }^{1}$ The ratio of plasma volume to BW usually decreases with increasing severity of obesity, ${ }^{7}$ as increased BW is accounted for by more fatty tissue, which contains less blood volume than muscle of the same weight. ${ }^{6}$ Overweight/obese individuals, therefore, typically have a lower plasma volume per kilogram of BW, while underweight individuals have a higher plasma volume per kilogram of BW than normal-weight individuals. Hence, weight-based dosing of FVIII would introduce a systematic error of unknown magnitude and may not result in the desired FVIII plasma levels in people with varying BW and/or body compositions. It has been suggested that dosing should consider fat mass as well as BW. ${ }^{2}$

Few studies have investigated the impact of different morphometric parameters on FVIII pharmacokinetics (PK), ${ }^{8-12}$ and clinical guidance for the dosing of FVIII in patients with haemophilia according to body composition is lacking. Data from a PK trial with the recombinant FVIII (rFVIII) turoctocog alfa (NovoEight, Novo Nordisk, Bagsværd, Denmark) in a small subset of patients with a body mass index $(B M I) \geq 30 \mathrm{~kg} / \mathrm{m}^{2}$ indicated that typical weight-based dosing leads to higher post-dose FVIII levels in overweight/obese (vs. normal-weight) patients. ${ }^{13}$

Here, we report the findings of a larger PK trial that assessed whether a relationship between PK (particularly recovery) and body composition could be identified and utilised to improve the predictability of plasma FVIII activity after treatment with turoctocog alfa in a dosing model.

\section{Materials and Methods}

\section{Trial Objectives and Endpoints}

The primary objective of the trial was to evaluate the singledose PK (particularly recovery) of turoctocog alfa in relation to BMI in patients with severe haemophilia A. Secondary objectives were to evaluate the single-dose PK of turoctocog alfa in relation to other BW and composition variables as potential PK predictors, and to add to the established safety record of turoctocog alfa.

The primary PK endpoint was FVIII activity at 30 minutes $\left(\mathrm{C}_{30 \mathrm{~min}}\right)$. Key secondary PK endpoints comprised incremental recovery at 30 minutes ( $\left.I_{30 \mathrm{~min}}\right)$, area under the FVIII activity time curve from 0 to infinity $\left(\mathrm{AUC}_{0-\mathrm{inf}}\right)$, terminal half-life $\left(t_{1 / 2}\right)$, clearance per kilogram of $B W(C L)$, and apparent volume of distribution per kilogram of BW at steady state $\left(\mathrm{V}_{\mathrm{ss}}\right)$ (-Supplementary Material, available in the online version). The safety endpoint was the number of adverse events (AEs) up to day 28.

\section{Patients}

Male patients aged $\geq 18$ years with severe haemophilia A(FVIII activity $<1 \%$ ) and $>150$ exposure days to FVIII products were included. Patients with a current/past history of FVIII inhibitors ( $\geq 0.6$ Bethesda Units) or any known coagulation disorder (apart from haemophilia A) were excluded. At visit 1, BMI was calculated based on BW and height. Patients were then grouped into five BMI categories: underweight $\left(<18.5 \mathrm{~kg} / \mathrm{m}^{2}\right)$, normal $\left(18.5-24.9 \mathrm{~kg} / \mathrm{m}^{2}\right)$, overweight $\left(25-29.9 \mathrm{~kg} / \mathrm{m}^{2}\right)$, obese class I $\left(30-34.9 \mathrm{~kg} / \mathrm{m}^{2}\right)$, or obese class II/III $\left(\geq 35 \mathrm{~kg} / \mathrm{m}^{2}\right)$. Recruitment was stratified by BMI group to ensure a balance between the five categories.

\section{Trial Design}

The PK trial was a multi-national, multi-centre, open-label, single-arm trial investigating the single-dose PK of turoctocog alfa in relation to BMI in previously treated adults with severe haemophilia A without inhibitors. The trial was conducted between October 2016 and June 2017 at 13 sites in seven countries (Austria, Bulgaria, Germany, Serbia, Spain, Taiwan and the United States).

Turoctocog alfa was supplied as a sterile, freeze-dried powder in single-use vials and reconstituted in $4.3 \mathrm{~mL}$ of $0.9 \%$ sodium chloride prior to intravenous injection. Total trial duration for each patient was 28 days, excluding a screening period of $\leq 28$ days. The trial included one PK session per patient. There were six planned visits: one 
screening visit (visit 1); one 72-hour PK session with four visits (visits 2-5); one follow-up visit (visit 6) that took place $28 \pm 7$ days after visit 2 . At visit 2 , each patient received a single intravenous bolus injection of turoctocog alfa at $50 \mathrm{IU}$ per kilogram of BW in a non-bleeding state after $\mathrm{a} \geq 96$-hour washout period for FVIII-containing products. PK sampling was performed 11 times between -1 hour (i.e., pre-dose) and 72 hours.

The trial was approved by independent ethics committees and institutional review boards, and was conducted in accordance with the Declaration of Helsinki ${ }^{14}$ and Good Clinical Practice guidelines. ${ }^{15}$ Written informed consent was obtained from all patients prior to any trial-related activity. The trial (NCT02941354) was registered at www. clinicaltrials.gov.

\section{Pharmacokinetic Assessments}

Blood samples for PK analysis were collected pre-dose, at 15 and 30 minutes and 1, 3, 6, 9, 24, 28, 48 and 72 hours post-dose. PK endpoints were calculated using FVIII activity and measured by one-stage clotting (activated partial thromboplastin reagent, SynthASil [Instrumentation Laboratory, Milan, Italy]) and chromogenic (Coamatic FVIII kit [Instrumentation Laboratory]) assays performed on a Coasys Plus $C$ analyser (Roche Diagnostics, Mannheim, Germany). ${ }^{16}$ Human plasma standard, calibrated against World Health Organization international FVIII standards, was used to calibrate both assays. All PK-derived parameters were determined using non-compartmental methods, as such methods are consistent with previous studies, ${ }^{16,17}$ simple to use and require no assumptions to be made.

\section{Measures of Body Composition and PK Predictors}

Measurements of body composition and other potential predictors (ideal BW [IBW], body surface area, lean BW, adjusted BW based on fat-free mass measurements from bioimpedance analysis [BIA], predicted blood volume) were investigated for their association with PK parameters based on the chromogenic assay.

Measurements of body composition: Body composition was measured at either visit 3, 4 or 5 using dual-energy Xray absorptiometry (DXA) and BIA. Where possible, both measurements were taken at the same visit. The whole-body DXA scan, conducted at a local imaging site, measured total fat mass, lean body mass and body fat percentage. BIA, conducted at the trial site using a Tanita DC 430 SMA Body Composition Analyzer (Tanita Corporation, Tokyo, Japan) supplied by Novo Nordisk, measured total fat mass, fat-free mass and body fat percentage. The average of two measurements was used for each parameter to reduce variability.

In addition to the above measurements, body composition variables were calculated using formulae based on BW and/or height measurements (-Table $\mathbf{1}$ ).

Relationship between coagulation parameters and BMI: The influence of BMI on different coagulation parameters, namely pro-thrombin time, pro-thrombin fragment $1+2$, von Willebrand factor (vWF), plasminogen activator inhibitor-1 (PAI-1) and plasmin-a2-antiplasmin (PAP) complex, was investigated.
Table 1 Formulae for key calculated predictors

\begin{tabular}{|l|l|}
\hline Parameter & Formula \\
\hline $\mathrm{BMI}\left(\mathrm{kg} / \mathrm{m}^{2}\right)$ & $\mathrm{BMI}=$ weight $(\mathrm{kg}) /[\text { height }(\mathrm{m})]^{2}$ \\
\hline $\mathrm{IBW}(\mathrm{kg})^{2}$ & $\mathrm{IBW}=$ height $(\mathrm{cm})-100-[$ height $(\mathrm{cm})-150] / 4$ \\
\hline $\mathrm{BSA}\left(\mathrm{m}^{2}\right)^{18}$ & $\mathrm{BSA}=\mathrm{BW}^{0.425} \times$ height in $\mathrm{cm}^{0.725} \times 0.007184$ \\
\hline $\mathrm{LBW}(\mathrm{kg})^{19}$ & $\mathrm{LBW}=(9,270 \times \mathrm{BW}) /(6,680+[216 \times \mathrm{BMI}])$ \\
\hline $\begin{array}{l}\mathrm{ABW}(\mathrm{kg}), \\
\text { based on FFM } \\
\text { measurements } \\
\text { from BIA }\end{array}$ & $\mathrm{ABW}=\mathrm{FFM}+40 \%(\mathrm{BW}-\mathrm{FFM})$ \\
\hline $\begin{array}{l}\text { PBV }(\mathrm{L}), \text { based } \\
\text { on indexed } \\
\text { blood } \\
\text { volume }\end{array}$ & $\mathrm{PBV}={ }_{\mathrm{n}} \mathrm{BV} \times \mathrm{BW} / 1,000$ \\
\hline
\end{tabular}

Abbreviations: ABW, adjusted body weight; BIA, bioimpedance analysis; BMI, body mass index; BSA, body surface area; BW, body weight; BV, blood volume; FFM, fat-free mass; IBW, ideal body weight; LBW, lean body weight; PBV, predicted blood volume.

Relationship between $t_{1 / 2}$, blood group and $v W F$ : The relationship between $\mathrm{t}_{1 / 2}$, blood group and vWF was also determined.

\section{Statistical Methods}

There were no formal statistical power calculations with respect to sample size. Thirty-five patients were planned to be included in the trial so that $6 \pm 1$ patients could be included in each of the five BMI categories at baseline. All dosed patients with data after dosing were included in the full and safety analysis sets.

Primary analysis comprised a linear regression of the primary endpoint $\left(\mathrm{C}_{30 \mathrm{~min}}\right)$ against BMI (BMI was treated as a continuous variable). Alternative parametric functional models for $\mathrm{C}_{30 \mathrm{~min}}$ were also explored as part of the primary analysis, covering quadratic and linear regression on logarithm-transformed predictors. No formal hypotheses were tested; however, linearity was determined by fitting linear, quadratic and loglinear models, and comparing the $R^{2}$ for these models to assess goodness-of-fit; $p$-values assessed statistical significance. Other conditions of validity (homoscedasticity and outlier assessment) were also evaluated by visual inspection. $C_{30 \text { min }}$ was also analysed using measured and calculated predictors in the regression analyses. Measured predictors were body fat percentage (measured by DXA and BIA), lean body mass (measured by DXA), fat-free mass (measured by BIA) and total body fat (measured by DXA and BIA). The mean of two measurements was used for all BIA assessments, unless only one was available. Calculated predictors comprised IBW, body surface area, lean $B W$, adjusted $B W$ and predicted blood volume. A selection of the analyses conducted for the primary endpoint $\left(C_{30 \mathrm{~min}}\right)$ was also conducted for the secondary PK endpoints ( $\mathrm{IR}_{30 \mathrm{~min}}, \mathrm{AUC}_{0 \text {-inf, }} \mathrm{t}_{1 / 2}$, $C L$ and $V_{s s}$; all analyses with $t_{1 / 2}$ were adjusted for age and blood group (O and Non-O). Non-compartmental analysis of PK data was performed using SAS (version 9.4; SAS Institute Inc., Cary, North Carolina, United States), using code made to mimic algorithms used in Phoenix WinNonlin (Certara, Princeton, New Jersey, United States).

All AEs were summarised and listed. 


\section{Dosing Model}

The required FVIII dose for on-demand treatment is currently determined using the following formula: dose $=\mathrm{BW}$ $(\mathrm{kg}) \times 100$ (as desired level is $1 \mathrm{IU} / \mathrm{mL}$ ) $\times 0.5{ }^{21,22}$ This formula indicates that, for a patient to have a $\mathrm{C}_{30 \mathrm{~min}}$ of $1 \mathrm{IU} / \mathrm{mL}$ at complete washout $(1 \mathrm{IU} / \mathrm{mL}$ relates to $100 \%$ FVIII activity [i.e., normal levels]), the dose should be 50 IU per kilogram of BW. The proposed new dosing model derived from the results of the current trial used $C_{30 \min }$ (as the goal was to achieve similar peak FVIII activity levels across BMI categories) and BMI, because the regression for BMI gave the largest $R^{2}$ values among the tested predictors; thus, a model using BMI has the largest potential to reduce variability in $\mathrm{C}_{30 \mathrm{~min}}$. Therefore, the new dosing model was based on linear regression of FVIII activity at 30 minutes, measured using the one-stage clotting assay, with BMI as a continuous predictor. The one-stage clotting assay was used because it is more commonly used for routine FVIII monitoring. The dosing model used the following formula:

$$
\begin{aligned}
\text { Optimal dose }= & \text { desired FVIII activity } \\
& \text { increase }(\%) \times M \times \mathrm{BW}
\end{aligned}
$$

where ' $M$ ' is the correction factor for dosing that is expected to provide similar $\mathrm{C}_{30 \mathrm{~min}}$ across patients with different BMI. ' $M$ ' was in the following form:

$$
0.5 /(\tilde{a}+\tilde{b} X)
$$

where $X$ is the average BMI in this trial for a given BMI category and $\tilde{a}+\tilde{b}$ were the intercept and slope parameter estimates obtained from a linear regression analysis of $\mathrm{C}_{30 \mathrm{~min}}$ against BMI and scaled so that a desired FVIII rise of $100 \%$ corresponded to the average FVIII activity in the normal BMI category. Further details of how linear regression was used to calculate the correction factor (' $M$ ') and thus establish the new dosing model are provided in the -Supplementary Material (available in the online version).

The reference value for $C_{30 \mathrm{~min}}$ (based on the one-stage clotting assay) was the mean value across all normal-weight patients in the trial. With this, it was intended that the proposed dosing model would give similar $C_{30 \min }$ values after dosing with turoctocog alfa across all BMI categories and irrespective of other descriptors of body composition in patients with haemophilia A. The dosing model was assessed by scaling the original data according to the new dosing model (- Supplementary Material, available in the online version) and re-analysing the relationship between PK and body measurements.

\section{Results}

\section{Patients}

Thirty-five patients received a single dose of turoctocog alfa. The overall mean age was 37.4 years (range: $23.0-57.0$ years); mean ages were similar across all BMI categories. The majority of patients were white $(N=32$ [91.4\%]). Patient demographics and baseline characteristics are shown in -Table 2.

\section{Pharmacokinetics}

FVIII activity: FVIII activity profiles showed an expected exponential decline over time with a clear trend of increasing FVIII activity levels from underweight to obese class II/III patients. The summary of key PK endpoints (based on the chromogenic assay) by BMI category is presented in - Table 3.

\section{Association of $C_{30 \mathrm{~min}}$ and $A U C_{0 \text {-inf }}$ with Measurements of Body Composition}

$\mathrm{C}_{30 \mathrm{~min}}$, the primary PK endpoint, was found to have a significant positive linear association with measurements of body composition, namely body fat percentage, lean body mass, fat-free mass and total fat mass (all measured by DXA and BIA) ( - Supplementary Table S1, available in the online version). Chromogenic and one-stage clotting assays yielded similar results in the statistical analyses of dependencies. While all measurements were significant predictors of $C_{30 \mathrm{~min}}$, none had a higher $R^{2}$ value (indicating how predictive each association is) than BMI, suggesting that BMI is the best predictor of PK endpoints ( - Fig. 1A). The $R^{2}$ values associated with BIA measurements tended to be higher than those associated with DXA measurements, suggesting that BIA measures give better prediction of PK endpoints than DXA. A statistically significant positive association between $\mathrm{AUC}_{0 \text {-inf }}$ and $\mathrm{BMI}$ was also observed ( - Fig. 1B), as well as with several other measurements (-Supplementary Table S1, available in the online version). Quadratic- and logarithm-transformed models did not add any additional information beyond that provided by the linear model. The association of other PK endpoints with measured predictors is provided in the -Supplementary Material and -Supplementary Table $\mathbf{5 1}$ (available in the online version).

\section{Association of PK Endpoints with BMI}

All results in this section are based on FVIII measurements derived from the chromogenic assay. Similar results for the primary and secondary endpoints were obtained when using the one-stage clotting assay.

$C_{30 \text { min }}$ : A statistically significant positive association between $\mathrm{C}_{30 \mathrm{~min}}$ and $\mathrm{BMI}$, in which $\mathrm{C}_{30 \mathrm{~min}}$ values increased with increasing BMI, was observed. The geometric mean $\mathrm{C}_{30 \mathrm{~min}}$ values ranged from $1.24 \mathrm{IU} / \mathrm{mL}$ (coefficient of variation [CV]: $17.3 \%$ ) in the underweight group to $1.96 \mathrm{IU} / \mathrm{mL}$ (CV: $13.2 \%)$ in obese class II/III patients (-Table 3; - Fig. 2A).

$I R_{30 \min }$ : A statistically significant positive association between $\mathrm{IR}_{30 \mathrm{~min}}$ (derived from $\mathrm{C}_{30 \mathrm{~min}}$ ) and BMI was observed. The geometric mean $\mathrm{IR}_{30 \mathrm{~min}}$ increased from $0.022(\mathrm{IU} / \mathrm{mL}) /(\mathrm{IU} / \mathrm{kg})(\mathrm{CV}: 17.7 \%)$ in underweight patients to $0.035(\mathrm{IU} / \mathrm{mL}) /(\mathrm{IU} / \mathrm{kg})(\mathrm{CV}: 13.3 \%)$ in obese class $\mathrm{II} / \mathrm{III}$ patients (-Table 3; - Fig. 2B).

$A U C_{0-i n f}:$ A statistically significant positive association between $\mathrm{AUC}_{0 \text {-inf }}$ and BMI was also observed. The geometric mean $\mathrm{AUC}_{0 \text {-inf }}$ values ranged from $17.8 \mathrm{IU} \times \mathrm{h} / \mathrm{mL}(\mathrm{CV}: 32.5 \%)$ in underweight patients to $29.7 \mathrm{IU} \times \mathrm{h} / \mathrm{mL}(\mathrm{CV}: 25.6 \%)$ in obese class II/III patients ( - Table 3; - Fig. 2C). 
Table 2 Patient demographics and baseline characteristics

\begin{tabular}{|c|c|c|c|c|c|c|}
\hline & \multicolumn{6}{|l|}{ BMI categories } \\
\hline & $\begin{array}{l}\text { Underweight } \\
<18.5 \mathrm{~kg} / \mathrm{m}^{2}\end{array}$ & $\begin{array}{l}\text { Normal weight } \\
18.5-24.9 \mathrm{~kg} / \mathrm{m}^{2}\end{array}$ & $\begin{array}{l}\text { Overweight } \\
25.0-29.9 \mathrm{~kg} / \mathrm{m}^{2}\end{array}$ & $\begin{array}{l}\text { Obese class I } \\
30.0-34.9 \mathrm{~kg} / \mathrm{m}^{2}\end{array}$ & $\begin{array}{l}\text { Obese class II/III } \\
\geq 35.0 \mathrm{~kg} / \mathrm{m}^{2}\end{array}$ & Total \\
\hline N & 5 & 7 & 9 & 7 & 7 & 35 \\
\hline \multicolumn{7}{|l|}{ Age $(y)$} \\
\hline Mean (SD) & $34.0(8.9)$ & $42.4(11.0)$ & $34.4(11.4)$ & $35.9(10.6)$ & $40.3(8.1)$ & $37.4(10.2)$ \\
\hline \multicolumn{7}{|l|}{ Race, $N(\%)$} \\
\hline White & $5(100.0)$ & $5(71.4)$ & $8(88.9)$ & $7(100.0)$ & $7(100.0)$ & $32(91.4)$ \\
\hline Asian & $0(0.0)$ & $1(14.3)$ & $1(11.1)$ & $0(0.0)$ & $0(0.0)$ & $2(5.7)$ \\
\hline Black or African American & $0(0.0)$ & $1(14.3)$ & $0(0.0)$ & $0(0.0)$ & $0(0.0)$ & $1(2.9)$ \\
\hline \multicolumn{7}{|l|}{ Height (m) } \\
\hline Mean (SD) & $1.81(0.07)$ & $1.70(0.09)$ & $1.74(0.10)$ & $1.76(0.10)$ & $1.78(0.06)$ & $1.76(0.09)$ \\
\hline \multicolumn{7}{|l|}{ BW (kg) } \\
\hline Mean (SD) & $55.26(5.86)$ & $63.14(5.95)$ & $82.98(12.5)$ & $102.3(12.7)$ & $131.9(17.4)$ & $88.69(29.3)$ \\
\hline \multicolumn{7}{|l|}{ BMI $\left(\mathrm{kg} / \mathrm{m}^{2}\right)$} \\
\hline Mean (SD) & $16.78(0.77)$ & $21.77(1.61)$ & $27.12(1.78)$ & $33.06(2.04)$ & $41.41(4.86)$ & $28.62(8.65)$ \\
\hline \multicolumn{7}{|l|}{ BFP (\%, DXA) } \\
\hline Mean (SD) & $17.2(6.4)$ & $27.1(8.7)$ & $28.9(8.3)$ & $35.1(6.9)$ & $44.7(8.7)$ & $30.9(11.3)$ \\
\hline \multicolumn{7}{|l|}{$\operatorname{BFP}(\%, \mathrm{BIA})$} \\
\hline Mean (SD) & $14.1(6.4)$ & $23.2(5.2)$ & $25.2(3.6)$ & $32.2(3.0)$ & $42.1(3.8)$ & $27.7(9.8)$ \\
\hline \multicolumn{7}{|l|}{$\mathrm{IBW}^{\mathrm{a}}(\mathrm{kg})$} \\
\hline Mean (SD) & $73.4(5.01)$ & $65.3(7.11)$ & $68.3(7.40)$ & $69.3(7.50)$ & $71.3(4.72)$ & $69.2(6.74)$ \\
\hline \multicolumn{7}{|l|}{$\mathrm{BSA}^{\mathrm{a}}\left(\mathrm{m}^{2}\right)$} \\
\hline Mean (SD) & $1.7(0.12)$ & $1.7(0.13)$ & $2.0(0.20)$ & $2.2(0.20)$ & $2.5(0.17)$ & $2.0(0.32)$ \\
\hline \multicolumn{7}{|l|}{$\mathrm{LBW}^{\mathrm{a}}(\mathrm{kg})$} \\
\hline Mean (SD) & $49.6(4.50)$ & $51.7(4.96)$ & $61.3(7.95)$ & $68.5(7.82)$ & 78.2 (6.09) & $62.5(12.10)$ \\
\hline \multicolumn{7}{|l|}{$A B W^{\mathrm{a}}(\mathrm{kg})$} \\
\hline Mean (SD) & $50.6(4.56)$ & $54.4(5.29)$ & $68.8(9.36)$ & $82.1(8.53)$ & $101.0(12.44)$ & 71.7 (19.68) \\
\hline \multicolumn{7}{|l|}{$\mathrm{PBV}^{\mathrm{a}}(\mathrm{L})$} \\
\hline Mean (SD) & $4.4(0.38)$ & $4.5(0.43)$ & $5.2(0.67)$ & $5.8(0.67)$ & $6.7(0.56)$ & $5.4(1.01)$ \\
\hline
\end{tabular}

Abbreviations: ABW, adjusted body weight; BFP, body fat percentage; BIA, bioimpedance analysis; BMI, body mass index; BSA, body surface area; BW, body weight; DXA, dual-energy X-ray absorptiometry; IBW, ideal body weight; LBW, lean body weight; PBV, predicted blood volume; SD, standard deviation. ${ }^{\text {a }}$ See $\mathbf{- T a b l e} \mathbf{1}$ for formulae for calculated predictors.

$t_{1 / 2}$ : The geometric mean ranged from 9.2 hours (CV: $\left.35.2 \%\right)$ to 11.5 hours (CV: $21.2 \%$ ) across the BMI categories ( - Table 3 ) and was found to be independent of BMI ( - Fig. 2D) and other predictors (-Supplementary Table S1, available in the online version). In addition, there was no relationship between BMI and the time to $1 \%$ of normal FVIII activity (-Supplementary Table S2 and - Supplementary Fig. S1 [available in the online version]).

$C L$ : Derived from $\mathrm{AUC}_{0-\text {-inf, }} \mathrm{CL}$ showed a significant negative association with $\mathrm{BMI}$, as expected. Underweight and obese class II/III groups showed a geometric mean CL of 3.16 (CV: $33.1 \%$ ) and $1.88 \mathrm{~mL} / \mathrm{h} / \mathrm{kg}$ (CV: $25.7 \%$ ), respectively (-Table 3; - Fig. 2E).

$V_{s s}$ : A significant negative association between $V_{s s}$ per kilogram of BW and BMI was observed. The highest geometric mean $\mathrm{V}_{\text {ss }}$ was found in the underweight group $(48.1 \mathrm{~mL} / \mathrm{kg}$ [CV: 22.3\%]) and the lowest in the obese class II/III patients (25.4 mL/kg [CV: 16.0\%]) (-Table 3; - Fig. 2F).

\section{Association of $\mathbf{C}_{\mathbf{3 0 m i n}}$ with Body Surface Area, Lean BW, Adjusted BW and Predicted Blood Volume}

We observed a significantly positive association of $C_{30 \text { min }}$ and derived parameter $I_{30 \mathrm{~min}}$ with most potential predictors of PK parameters, namely body surface area, lean BW, adjusted BW and predicted blood volume (-Supplementary Table S1, available in the online version). However, there was no significant association of $\mathrm{C}_{30 \mathrm{~min}}$ with $\operatorname{IBW}\left(p=0.8801 ; R^{2}\right.$ : $0.0007)$ (-Fig. 1C) and $\mathrm{AUC}_{0 \text {-inf }}$ with $\mathrm{IBW}\left(p=0.7706 ; R^{2}\right.$ : 0.0026 ) (-Fig. 1D). The $R^{2}$ values for PK predictors were all lower than that for BMI and most other body composition measurements. As with the assessments, quadratic- and logarithm-transformed models of calculated predictors did not add any additional information beyond that provided by the linear model. The association of other PK endpoints with body surface area, lean BW, adjusted BW and predicted blood volume is provided in the - Supplementary Material (available in the online version). 
Table 3 Summary of key PK parameters by BMI category (chromogenic assay)

\begin{tabular}{|c|c|c|c|c|c|}
\hline & \multicolumn{5}{|l|}{ BMI categories } \\
\hline & $\begin{array}{l}\text { Underweight } \\
<18.5 \mathrm{~kg} / \mathrm{m}^{2}\end{array}$ & $\begin{array}{l}\text { Normal weight } \\
18.5-24.9 \mathrm{~kg} / \mathrm{m}^{2}\end{array}$ & $\begin{array}{l}\text { Overweight } \\
25.0-29.9 \mathrm{~kg} / \mathrm{m}^{2}\end{array}$ & $\begin{array}{l}\text { Obese class I } \\
30.0-34.9 \mathrm{~kg} / \mathrm{m}^{2}\end{array}$ & $\begin{array}{l}\text { Obese class II/III } \\
\geq 35.0 \mathrm{~kg} / \mathrm{m}^{2}\end{array}$ \\
\hline N & 5 & 7 & 9 & 7 & 7 \\
\hline \multicolumn{6}{|l|}{$C_{30 \min }(I U / m L)$} \\
\hline Geometric mean (CV\%) & $1.24(17.3)$ & $1.65(9.4)$ & $1.66(15.9)$ & $1.79(15.1)$ & $1.96(13.2)$ \\
\hline Range & $1.02-1.58$ & $1.47-1.90$ & $1.32-2.02$ & $1.46-2.31$ & $1.59-2.28$ \\
\hline \multicolumn{6}{|l|}{$I_{30 \min }(I U / m L) /(I U / k g)$} \\
\hline Geometric mean (CV\%) & $0.022(17.7)$ & $0.029(9.5)$ & $0.029(16.0)$ & $0.032(15.5)$ & $0.035(13.3)$ \\
\hline Range & $0.018-0.028$ & $0.026-0.034$ & $0.023-0.036$ & $0.026-0.041$ & $0.028-0.041$ \\
\hline \multicolumn{6}{|l|}{$\mathrm{AUC}_{0-\text { inf }}(\mathrm{IU} \times \mathrm{h} / \mathrm{mL})$} \\
\hline Geometric mean (CV\%) & $17.8(32.5)$ & $26.1(33.6)$ & $21.3(44.1)$ & $24.9(58.0)$ & $29.7(25.6)$ \\
\hline Range & $14.0-30.9$ & $17.1-40.4$ & $13.3-39.0$ & $11.0-41.1$ & $19.0-42.4$ \\
\hline \multicolumn{6}{|l|}{$t_{1 / 2}(h)$} \\
\hline Geometric mean (CV\%) & $11.5(21.2)$ & $10.9(38.5)$ & $9.2(35.2)$ & $9.9(38.1)$ & $10.3(21.7)$ \\
\hline Range & $8.3-15.0$ & $5.7-15.3$ & $5.8-15.5$ & $5.2-13.6$ & $8.3-15.4$ \\
\hline \multicolumn{6}{|l|}{$\mathrm{CL}(\mathrm{mL} / \mathrm{h} / \mathrm{kg})$} \\
\hline Geometric mean (CV\%) & $3.16(33.1)$ & $2.15(33.3)$ & $2.64(44.3)$ & $2.25(58.6)$ & $1.88(25.7)$ \\
\hline Range & $1.80-4.03$ & $1.37-3.30$ & $1.44-4.28$ & $1.36-5.17$ & $1.31-2.94$ \\
\hline \multicolumn{6}{|l|}{$\mathrm{V}_{\mathrm{ss}}(\mathrm{mL} / \mathrm{kg})$} \\
\hline Geometric mean (CV\%) & $48.1(22.3)$ & $31.0(15.0)$ & $31.1(18.6)$ & $28.5(17.9)$ & $25.4(16.0)$ \\
\hline Range & $35.3-61.1$ & $24.0-36.9$ & $23.0-41.1$ & $22.3-37.3$ & $19.1-32.5$ \\
\hline
\end{tabular}

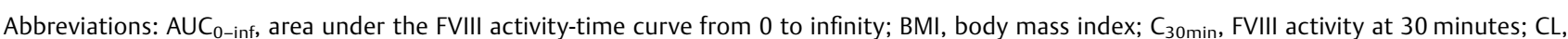
clearance; CV, coefficient of variation; $\mathbb{R}_{30 \mathrm{~min}}$, incremental recovery at 30 minutes; PK, pharmacokinetics; $S D$, standard deviation; $t_{1 / 2}$, terminal halflife; $V_{s s}$, apparent volume of distribution at steady state.

\section{Coagulation Parameters and BMI}

We found no association between coagulation parameters (i.e., pro-thrombin time, pro-thrombin fragment $1+2$, vWF and PAP [data not shown]) and BMI. However, increased levels of PAI-1 in obese class II/III patients were observed.

\section{Relationship between $\mathbf{t}_{1 / 2}$, Blood Group and vWF}

We observed a trend of decreasing $t_{1 / 2}$ with decreasing $v W F$ antigen levels, with a large variation in $t_{1 / 2}$ and vWF levels (-Fig. 3A). Additionally, patients with blood group $O$ tended to have a shorter $t_{1 / 2}$ than patients with other blood groups (-Fig. 3B). Adjusting for BMI did not change the significant influence of $v W F$ on $t_{1 / 2}$ (data not shown).

\section{Dosing Model}

The above PK results were used to derive a new dosing model showing the potential for dose adjustments that would produce more uniform $\mathrm{C}_{30 \mathrm{~min}}$ values across BMI categories. The dosing model used the following formula:

$$
\begin{aligned}
& \text { Optimal dose }= \text { desired FVIII activity } \\
& \text { increase }(\%) \times M \times \mathrm{BW}
\end{aligned}
$$

where ' $M$ ' is the correction factor for dosing that is expected to provide similar $C_{30 \mathrm{~min}}$ across patients with different BMI:
- Underweight: 0.55

- Normal weight: 0.51

- Overweight: 0.47

- Obese class I: 0.43

- Obese class II/III: 0.39

Between-patient variation in $\mathrm{C}_{30 \mathrm{~min}}$ was reduced by $40.5 \%$, and between-patient variation for $\mathrm{AUC}_{0 \text {-inf }}$ was reduced by $26.4 \%$. The observed total dose based on an administered dose of 50 IU per kilogram of BW and the new total dose based on the model by BW and BMI category are shown in - Table 4, along with potential dosing adjustment based on the model; the mean total dose adjustment varied from +243.3 IU in underweight patients to -1489.6 IU in obese class II/III patients. None of the other investigated PK predictors further improved the dosing model.

\section{Safety}

No inhibitor development or AEs leading to death or withdrawal were reported, and no new safety concerns (including no adverse drug reactions) were observed.

\section{Discussion}

The current trial was conducted to investigate the singledose PK of the rFVIII turoctocog alfa in relation to BMI in 

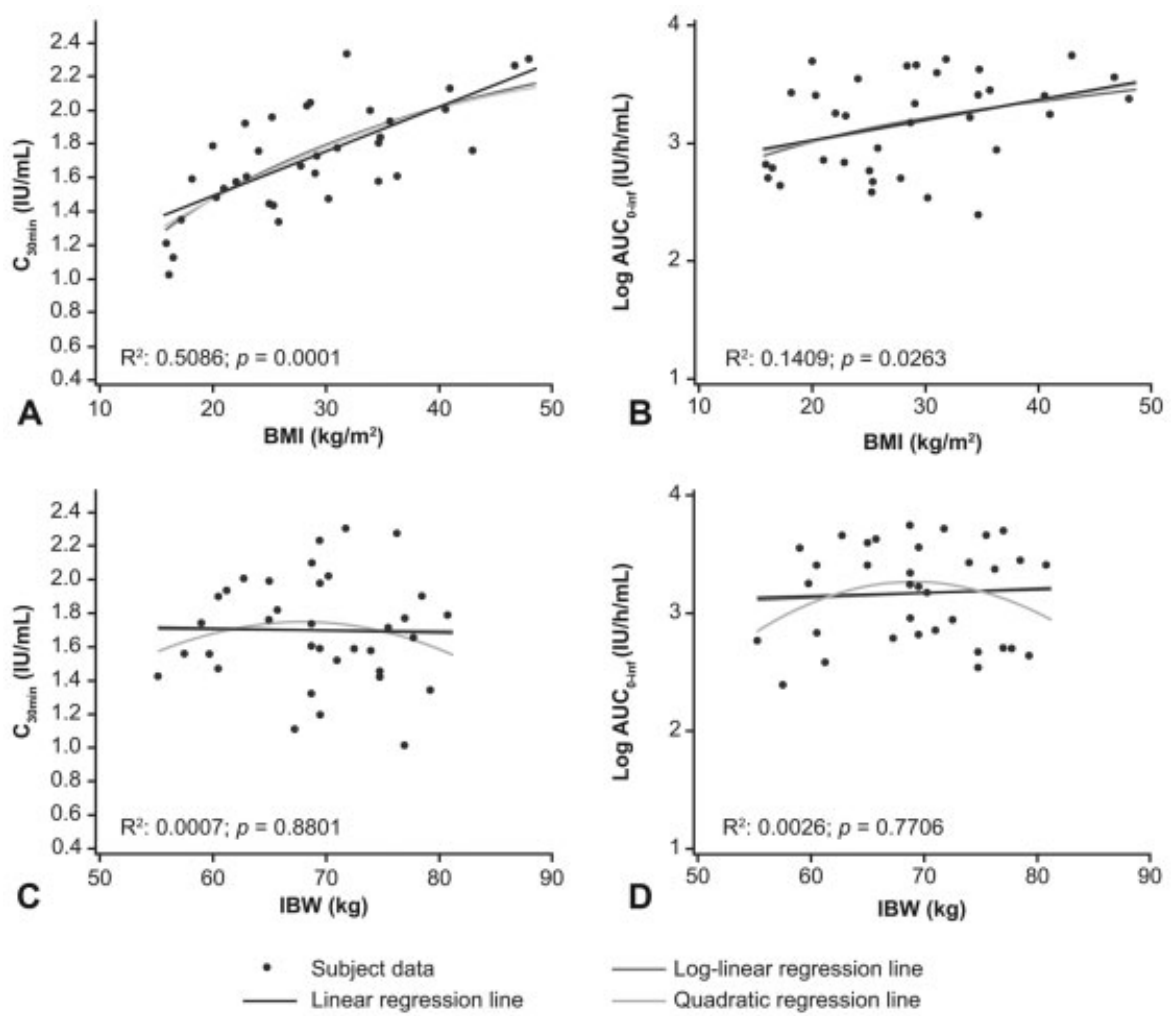

Fig. $1 C_{30 \mathrm{~min}}$ and $A U C_{0-\text { inf }}$ versus BMI and IBW (chromogenic assay). (A) $C_{30 \text { min }}$ showed significant positive associations with BMI (linear regression line, $p<0.0001 ; R^{2}: 0.5086$ ). (B) $A \cup C_{0-i n f}$ showed significant positive associations with $\mathrm{BMI}$ (linear regression line, $p<0.0263 ; R^{2}$ : 0.1409 ). (C) IBW was not associated with PK endpoints; linear regression line, $p=0.8801$ for $C_{30 \mathrm{~min}} ; R^{2}, 0.0007$ is from the linear regression

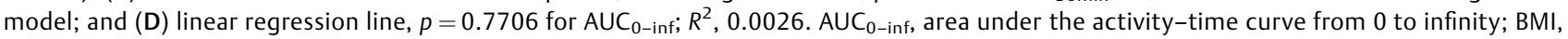
body mass index; $\mathrm{C}_{30 \mathrm{~min}}$, FVIII activity at 30 minutes; IBW, ideal body weight; PK, pharmacokinetic.

previously treated adults with severe haemophilia $\mathrm{A}$, with a focus on recovery. Overall, the trial showed increased $C_{30 \mathrm{~min}}$, $\mathrm{AUC}_{0 \text {-inf }}$ and $\mathrm{IR}_{30 \mathrm{~min}}$ with increasing BMI, while $\mathrm{CL}$ and $\mathrm{V}_{\mathrm{ss}}$ decreased with increasing BMI. Of all the measured and calculated predictors assessed (IBW, body surface area, lean BW, adjusted BW and predicted blood volume), BMI was the most robust and best predictor of PK endpoints. The exception to this was $t_{1 / 2}$, which seemed to be independent of all predictors.

\section{The Dosing Model}

The combined BW and BMI dosing model resulted in predictable PK parameters in patients with different body compositions. The innovative component of the dosing model was incorporation of the correction factor ' $\mathrm{M}$ ' to calculate dosage adjustments based on the difference in $\mathrm{C}_{30 \mathrm{~min}}$ across five BMI categories. The model reduced total variation by $40.5 \%$ for $\mathrm{C}_{30 \mathrm{~min}}$ and by $26.4 \%$ for $\mathrm{AUC}_{0 \text {-inf. }}$. No other predictor significantly improved the dosing model beyond the addition of BMI (data not shown). The new dosing model also indicates that, when using current standard dose calculations, underweight patients might receive an insufficient FVIII dose for effective bleed control, overweight/obese patients might receive more FVIII than needed and normal-weight patients would require little or no dose adjustment compared with the current dosing recommendation (-Table 4).

\section{Why BMI Performs Well as a Predictor of FVIII PK}

IBW has been suggested instead of actual BW for FVIII dose calculations. ${ }^{10,11}$ A recent study performed on simulated patients found that dosing based on IBW was cost effective and provided the highest proportion of time spent above $1 \%$ FVIII on standard prophylaxis. ${ }^{3}$ In contrast, in the current trial, which included real patients and measured PK parameters, IBW was not found to be a useful metric for FVIII dose adjustment when the goal is to achieve uniform PK across weight categories. IBW is directly correlated to height and is not based on any aspect of body composition or weight. In the current trial, IBW was the only predictor that did not show any association with any PK endpoints. A possible explanation for BMI being a good predictor of PK endpoints might be that the trial participants had body compositions (i.e., proportions of highly vascularised, lean tissue vs. poorly vascularised, fatty tissue) that were typical of their BMI category. In this way, some fatty tissues are accounted for by BMI, whereas IBW would completely ignore the fatty tissue and thereby over-adjust the dose. FVIII recovery has been found to increase with increasing BMI and BW..$^{9,11,23} \mathrm{~A}$ regression-tree analysis conducted by Henrard et al to examine the impact of various morphometric parameters (different parameters to those chosen in the current trial) on FVIII recovery among 201 adults ( $>18$ years) with haemophilia A found BMI to be the strongest predictor of FVIII 

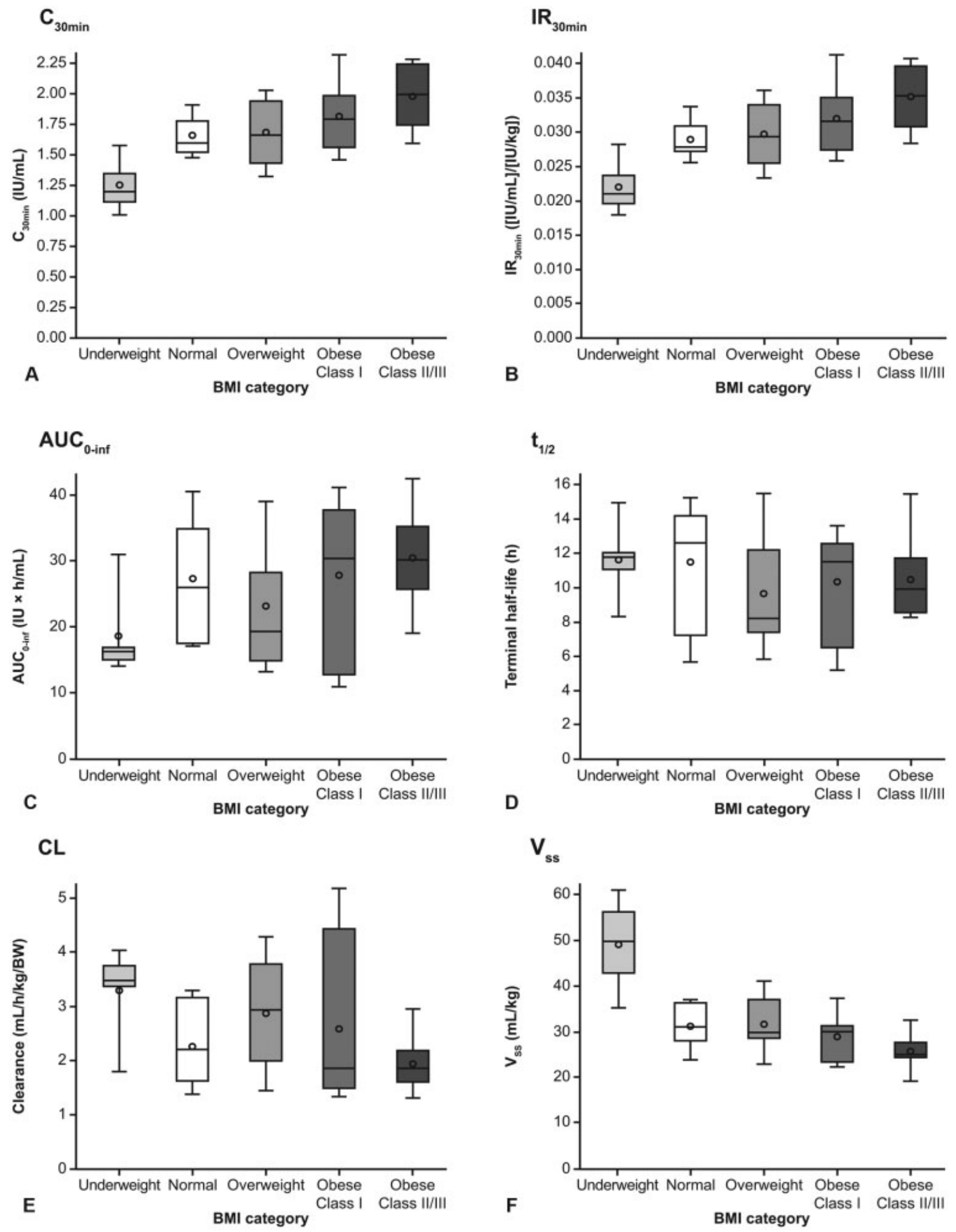

Fig. $2 C_{30 \min }, I_{30 \min }, A \cup C_{0-i n f}, t_{1 / 2}, C L$ and $V_{s s}$ by BMI category (chromogenic assay). Statistically significant positive association between BMI and (A) $C_{30 \mathrm{~min}}$, (B) $\mathbb{I}_{30 \mathrm{~min}}$ and (C) $A \cup C_{0 \text {-inf; }}$ lack of association between BMI and (D) $t_{1 / 2}$; statistically significant negative association between BMI and $(E) C L$ and $(F) V_{s s}$. The box and whisker plots are based on standard non-parametric measures, and the circles represent arithmetic means. Arithmetic mean $C_{30 \min }$ ranged from 1.25 to $1.98 \mathrm{IU} / \mathrm{mL}$ in underweight to obese class II/III patients, respectively. Individual $C_{30 \mathrm{~min}}$ ranged from 1.02 to $2.31 \mathrm{IU} / \mathrm{mL}$. BMI, body mass index; BW, body weight; $C_{30 \mathrm{~min}}$, FVIII activity at 30 minutes; $I R_{30 \mathrm{~min}}$, incremental recovery at 30 minutes; $\mathrm{V}_{\mathrm{ss}}$, apparent volume of distribution per kilogram of BW at steady state. BMI categories are derived from BMI calculated at screening: underweight $\left(\mathrm{BMI}<18.5 \mathrm{~kg} / \mathrm{m}^{2}\right)$, normal range (BMI: $\left.18.5-24.9 \mathrm{~kg} / \mathrm{m}^{2}\right)$, overweight (BMI: $\left.25-29.9 \mathrm{~kg} / \mathrm{m}^{2}\right)$, obese class I (BMI $\left.30-34.9 \mathrm{~kg} / \mathrm{m}^{2}\right)$ and obese class II $/$ III $\left(B M I \geq 35 \mathrm{~kg} / \mathrm{m}^{2}\right)$. 

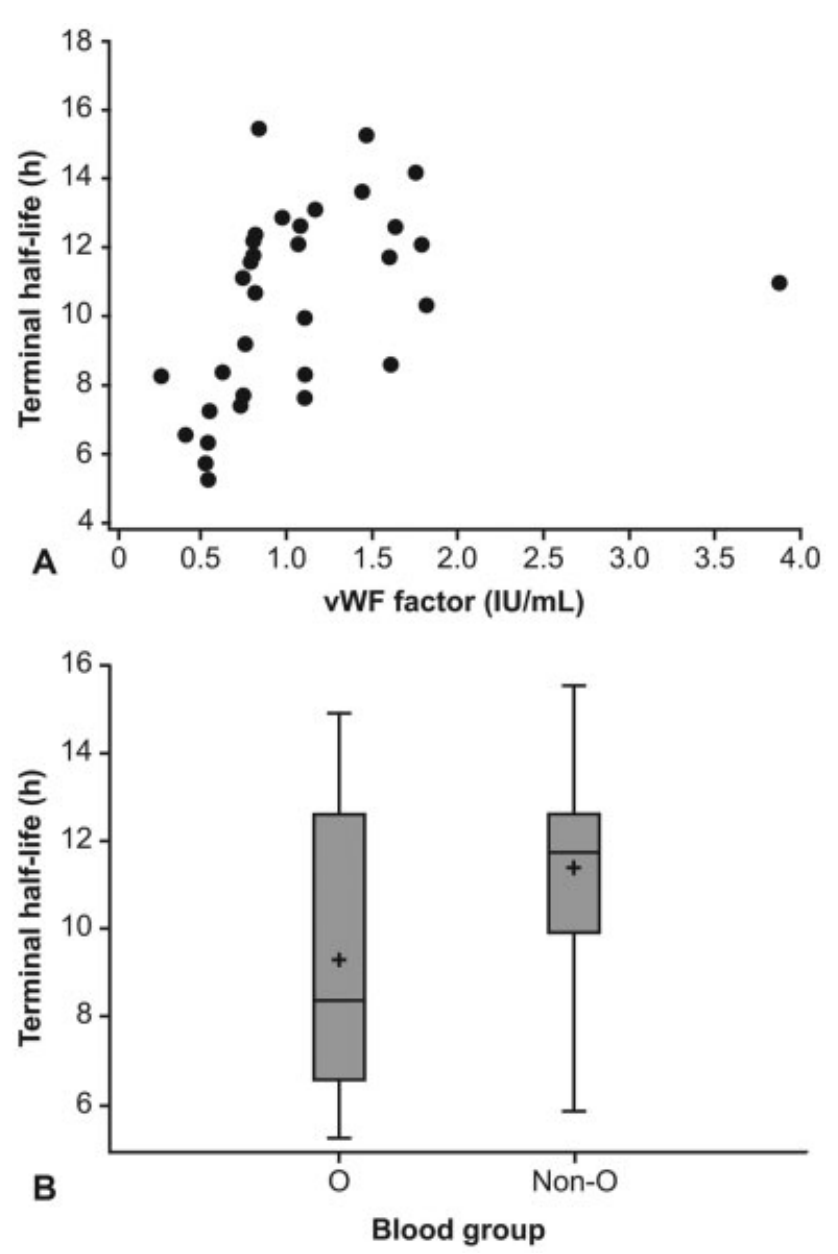

Fig. $3 t_{1 / 2}$ versus vWF (A) and blood group (B) (chromogenic assay) $t_{1 / 2}$ versus blood group is a box plot $(\mathrm{B})$, ' + ' represents the mean and the horizontal lines within the box represents the median. $t_{1 / 2}$, terminal half-life; vWF, von Willebrand factor. recovery, in line with current findings. ${ }^{11}$ The regression-tree analysis also found significantly different $(p<0.001)$ median FVIII recovery values of $1.60,2.14$ and 2.70 among patients with $\mathrm{BMI}<20.3,20.3$ to 29.5 and $\geq 29.6 \mathrm{~kg} / \mathrm{m}^{2}$, respectively.

\section{Exploring the Measures of Body Composition}

Although DXA can measure fat and muscle compartments, ${ }^{24,25}$ BIA body composition measurements gave slightly better predictions $\left(R^{2}\right)$ of PK parameters than DXA measurements in the current trial, which is unexpected. As FVIII activity is primarily distributed in blood, the BIA assessment may have performed better than DXA due to the dependency of BIA on the body's fluid content (including blood). BIA measurements can also be affected by factors such as physical activity, hydration status and consumption of food and beverages. ${ }^{24}$ To limit the interference of these factors in the current trial, patients were advised to drink fluid normally during the 24 hours prior to the scan $(\sim 2 \mathrm{~L})$, avoid alcohol consumption (within 12 hours prior to the scan), avoid large meals (within 4-6 hours prior to the scan) and were asked to empty their bladder prior to the scan.

Measures of fat and lean mass directly obtained by DXA and BIA performed weaker as predictors of FVIII PK than the measurements of BMI. This may be explained by the fact that these measures account for lean or fatty tissue but not for both, while blood is unequally distributed in both tissues.

\section{Relationship of BMI to $t_{1 / 2}$, Blood Group and vWF}

Increased adipose tissues in obese patients might contribute to the release of adipokines and cytokines, resulting in changes in coagulation factor levels in the liver. ${ }^{26,27}$ Considering this, the influence of BMI on different coagulation parameters in the current trial was explored. However, no influence of BMI on coagulation parameters was seen, except increased PAI-1 levels in the obese class II/III patients versus

Table 4 Average total dose adjustment (M) across all BMI groups based on the proposed model when dosing turoctocog alfa at $50 \mathrm{IU} / \mathrm{kg}$

\begin{tabular}{|c|c|c|c|c|c|}
\hline & \multicolumn{5}{|l|}{ BMI categories } \\
\hline & $\begin{array}{l}\text { Underweight } \\
<18.5 \mathrm{~kg} / \mathrm{m}^{2}\end{array}$ & $\begin{array}{l}\text { Normal weight } \\
18.5-24.9 \mathrm{~kg} / \mathrm{m}^{2}\end{array}$ & $\begin{array}{l}\text { Overweight } \\
25.0-29.9 \mathrm{~kg} / \mathrm{m}^{2}\end{array}$ & $\begin{array}{l}\text { Obese class I } \\
30.0-34.9 \mathrm{~kg} / \mathrm{m}^{2}\end{array}$ & $\begin{array}{l}\text { Obese class II/III } \\
\geq 35.0 \mathrm{~kg} / \mathrm{m}^{2}\end{array}$ \\
\hline$N$ & 5 & 7 & 9 & 7 & 7 \\
\hline \multicolumn{6}{|l|}{ Observed dose (IU) } \\
\hline Mean dose (SD) & $2,780.2(277.4)$ & $3,214.1(279.8)$ & $4,201.7(622.5)$ & $5,144.1(639.2)$ & $6,642.9(838.1)$ \\
\hline \multicolumn{6}{|c|}{ New dose using dosing model } \\
\hline Mean dose (SD) & $3,023.5(282.5)$ & $3,216.3(302.9)$ & $3,890.9(515.7)$ & $4,424.5(508.4)$ & $5,153.3(429.8)$ \\
\hline \multicolumn{6}{|l|}{ Change from observed } \\
\hline Mean (SD) & $243.3(25.3)$ & $2.2(84.5)$ & $-310.7(126.4)$ & $-719.6(182.5)$ & $-1,489.6(469.1)$ \\
\hline Correction factor (M) & 0.55 & 0.51 & 0.47 & 0.43 & 0.39 \\
\hline
\end{tabular}

Abbreviations: $\mathrm{BMI}$, body mass index; $\mathrm{M}$, the correction factor to dosing that is expected to provide similar $\mathrm{C}_{30 \text { min }}$ across patients with different BMI (i.e., the factor for multiplication with body weight to obtain the dose that is predicted to give the same $C_{30 \mathrm{~min}}$ from the one-stage clotting assay as the average in the normal BMI category in this study); SD, standard deviation.

Note: Dosing model was based on linear regression results for one-stage clotting assay versus BMI. Results from the one-stage clotting assay were used for the dosing model as these seemed the most relevant, showing the greatest decrease in standard deviation (vs. results using the chromogenic assay). 
non-obese patients, consistent with a study that also found elevated PAI-1 levels in obese individuals, possibly indicating reduced fibrinolysis in this group. ${ }^{5}$

When investigating the relationship between $t_{1 / 2}$, blood group and vWF, we found a large variation of $t_{1 / 2}$ and $v W F$ antigen levels. Nevertheless, $t_{1 / 2}$ appeared to decrease with decreasing vWF levels, which would be expected based on the tight association between FVIII and vWF; vWF maintains FVIII stability and prevents its degradation and $\mathrm{CL}^{28}$ Patients with blood group $\mathrm{O}$ have lower vWF levels than those with other blood groups. ${ }^{29}$ As expected, patients with blood group $\mathrm{O}$ in the current trial had lower vWF levels and therefore a shorter $t_{1 / 2}$ than patients with other blood groups.

\section{Limitations}

The study showed an impact of BMI on peak levels after dosing, but not on $t_{1 / 2}$. Achieving certain peak levels is primarily relevant for managing surgery or acute bleeds in patients with haemophilia. Routine prophylaxis, however, targets certain trough levels that are mainly influenced by individual $t_{1 / 2}$. Therefore, our data, including the new dosing model, are not relevant for routine prophylaxis dosing.

Chromogenic and one-stage clotting assays yielded similar results in the PK assessments, although there was a shift in FVIII concentration values between the two assays. As the chromogenic assay provided more stable results and led to exclusion of fewer samples than the one-stage assay, results obtained with the chromogenic assay have been presented for PK analyses. However, results from the one-stage clotting assay (more commonly used for routine FVIII monitoring) were used for the dosing model as they showed the greatest decrease in standard deviation (vs. results using the chromogenic assay); hence, we presented results based on the one-stage assay for the dosing model.

Given the negative association observed between age and dose requirement in the literature, ${ }^{30}$ lack of adjustment for age in the current study may also be a potential limitation of our findings; age did not affect $\mathrm{C}_{30 \mathrm{~min}}$, $\mathrm{AUC}$ and $\mathrm{CL}$, but the range of ages in the current trial may have been too small to show differences.

Another limitation of the trial was that the study population did not include extremely underweight patients (for example, elderly individuals or those with low fat mass) or extremely muscular individuals (such as body builders and athletes) or severely anaemic patients (to factor haematocrit levels); hence, the results are only applicable to a population with similar attributes. There is a need to validate the model in all BMI populations. BMI may be a poorer predictor of FVIII PK in patients with differing body compositions (e.g., those with greater muscle mass). However, high BMI due to a high volume of muscle mass is rare in patients with severe haemophilia.

Finally, some studies found two-compartmental analysis superior to non-compartmental analysis (e.g., Björkman et $\mathrm{al}^{31}$ ), while others did not (e.g., Morfini et $\mathrm{al}^{32}$ ). For turocto$\operatorname{cog}$ alfa PK data, there was no clear distribution phase or divergent trend regarding observed PK overlaid with predicted PK based on non-compartmental methods. As such, non-compartmental analysis had a good fit for turoctocog alfa PK data.

\section{Conclusion}

The trial confirmed that the PK of FVIII $\left(C_{30 \text { min }}\right.$, AUC, IR ${ }_{30 m i n}$, $\mathrm{CL}$ and $\mathrm{V}_{\mathrm{SS}}$ ) depends on BMI and body composition. A statistically significant positive association between $C_{30 \text { min }}$ and BMI, and between $\mathrm{C}_{30 \text { min }}$ and body surface area, lean BW, adjusted BW and predicted blood volume, was observed. Furthermore, of the parameters assessed in the trial, BMI was found to be the best predictor of PK endpoints, except for $t_{1 / 2}$, which appears to be independent. We also propose a novel and simple dosing model, which allowed improved predictability of plasma FVIII activity after treatment with rFVIII across all BMI categories. The dosing model introduced a correction factor ' $\mathrm{M}$ ' for each BMI category ranging from 0.55 for underweight to 0.39 for obese class II/III. Future studies to explore the relationship between FVIII activity levels and clinical efficacy are warranted.

\section{What is known about this topic?}

- FVIII products are typically dosed on a per-kilogram basis according to total body weight.

- Therapeutic FVIII administration based solely on body weight leads to varying FVIII levels.

- Few studies have investigated the impact of different morphometric parameters on FVIII PK, and clinical guidance for the dosing of FVIII in patients with haemophilia according to body composition is lacking.

\section{What does this paper add?}

- Pharmacokinetics of recombinant FVIII administration in persons with varying BMI was assessed in a singledose PK trial.

- $C_{30 \text { min }}, I_{30 \text { min }}, A U_{0-\text { inf }}$ correlated with BMI, whereas $\mathrm{t}_{1 / 2}$ did not.

- Of several morphometric parameters assessed, BMI best predicted incremental recovery.

- Body weight- and BMI-based model allowed dosing with better FVIII predictability across BMI categories.

\section{Note}

All authors confirm they have had full access to the data and contributed to the drafting of this manuscript.

\section{Funding}

This work was funded by Novo Nordisk A/S (Bagsværd, Denmark). Novo Nordisk's policy on data sharing may be found at https://www.novonordisk-trials.com/how-access-clinical-trial-datasets.

\section{Conflict of Interest}

A.T. has received research support, honoraria or consultation fees from Alnylam, Bayer, Biogen Idec, Biotest, Boehringer Ingelheim, Bristol-Myers Squibb, CSL Behring, Leo 
Pharma, Novo Nordisk, Octapharma, Pfizer, Roche, Shire and SOBI. A.R.C. has received reimbursement for attending symposia/congresses and/or honoraria for speaking or consulting from Novo Nordisk and other companies. G.G. has received reimbursement for attending symposia/congresses and/or honoraria for speaking or consulting from Novo Nordisk and other companies. V.J.-Y. has received reimbursement for attending symposia/congresses and/or honoraria for speaking and/or honoraria for consulting, and/or funds for research from Bayer, CSL Behring, Grifols, Novo Nordisk, Octapharma, Pfizer, Roche, Shire and SOBI. M.P. is a paid consultant for Novo Nordisk A/S. T.L. has received investigator fees as a participant of the clinical trial from Novo Nordisk. M.M. has no conflicts of interest to declare. I.M. is an employee of Novo Nordisk A/S. P.M. has no conflicts of interest to declare. I.P. has received honoraria or consultation fees from Bayer, Biotest, CSL Behring, Novo Nordisk, Octapharma, Pfizer, Roche, Shire and SOBI; also research support from CSL Behring and Novo Nordisk. P.P. is an employee of Novo Nordisk A/S.

\section{Acknowledgments}

The authors would like to thank Lars Korsholm for his scientific advice and critical review of the manuscript. Medical writing support was provided by Jo Fetterman, $\mathrm{PhD}$ (Parexel, United Kingdom).

\section{References}

1 Björkman S, Berntorp E. Pharmacokinetics of coagulation factors: clinical relevance for patients with haemophilia. Clin Pharmacokinet 2001;40(11):815-832

2 Henrard S, Speybroeck N, Hermans C. Body weight and fat mass index as strong predictors of factor VIII in vivo recovery in adults with hemophilia A. J Thromb Haemost 2011;9(09):1784-1790

3 McEneny-King A, Chelle P, Henrard S, Hermans C, Iorio A, Edginton AN. Modeling of body weight metrics for effective and cost-efficient conventional factor VIII dosing in hemophilia A prophylaxis. Pharmaceutics 2017;9(04):E47

4 Young G. New challenges in hemophilia: long-term outcomes and complications. Hematology (Am Soc Hematol Educ Program) 2012;2012:362-368

5 Tuinenburg A, Biere-Rafi S, Peters M, et al. Obesity in haemophilia patients: effect on bleeding frequency, clotting factor concentrate usage, and haemostatic and fibrinolytic parameters. Haemophilia 2013;19(05):744-752

6 Wong TE, Majumdar S, Adams E, et al; Healthy Weight Working Group. Overweight and obesity in hemophilia: a systematic review of the literature. Am J Prev Med 2011;41(06, Suppl 4):S369-S375

7 Feldschuh J, Enson Y. Prediction of the normal blood volume. Relation of blood volume to body habitus. Circulation 1977;56(4 Pt 1):605-612

8 Björkman S, Blanchette VS, Fischer K, et al; Advate Clinical Program Group. Comparative pharmacokinetics of plasma- and albumin-free recombinant factor VIII in children and adults: the influence of blood sampling schedule on observed age-related differences and implications for dose tailoring. J Thromb Haemost 2010;8(04):730-736

9 Blanchette VS, Shapiro AD, Liesner RJ, et al; rAHF-PFM Clinical Study Group. Plasma and albumin-free recombinant factor VIII: pharmacokinetics, efficacy and safety in previously treated pediatric patients. J Thromb Haemost 2008;6(08):1319-1326
10 Collins PW, Fischer K, Morfini M, Blanchette VS, Björkman S; International Prophylaxis Study Group Pharmacokinetics Expert Working Group. Implications of coagulation factor VIII and IX pharmacokinetics in the prophylactic treatment of haemophilia. Haemophilia 2011;17(01):2-10

11 Henrard S, Speybroeck N, Hermans C. Impact of being underweight or overweight on factor VIII dosing in hemophilia $\mathrm{A}$ patients. Haematologica 2013;98(09):1481-1486

12 Kepa S, Horvath B, Reitter-Pfoertner S, et al. Parameters influencing FVIII pharmacokinetics in patients with severe and moderate haemophilia A. Haemophilia 2015;21(03):343-350

13 Zupančić-Šalek S, Ozelo MC, Korsholm L, et al. An evaluation of the pharmacokinetics of turoctocog alfa in relation to body mass index (BMI) in patients with haemophilia A (HA). Res Pract Thromb Haemost 2017;1(01):671

14 World Medical Association. World Medical Association Declaration of Helsinki: ethical principles for medical research involving human subjects. JAMA 2013;310(20):2191-2194

15 International Conference on Harmonisation of Technical Requirements for Registration of Pharmaceuticals for Human Use (ICH), Harmonised Tripartite Guideline: Guideline for Good Clinical Practice E6(R1); 1996. Available at: http://apps.who.int/medicinedocs/documents/s22154en/s22154en.pdf. Accessed November 17, 2019

16 Kulkarni R, Karim FA, Glamocanin S, et al. Results from a large multinational clinical trial (guardian ${ }^{\mathrm{TM}} 3$ ) using prophylactic treatment with turoctocog alfa in paediatric patients with severe haemophilia A: safety, efficacy and pharmacokinetics. Haemophilia 2013;19(05):698-705

17 Lentz SR, Misgav M, Ozelo M, et al. Results from a large multinational clinical trial (guardian ${ }^{\mathrm{TM}}$ 1) using prophylactic treatment with turoctocog alfa in adolescent and adult patients with severe haemophilia A: safety and efficacy. Haemophilia 2013;19(05): 691-697

18 Du Bois D, Du Bois EF. A formula to estimate the approximate surface area if height and weight be known. 1916. Nutrition 1989; 5(05):303-311

19 Janmahasatian S, Duffull SB, Ash S, Ward LC, Byrne NM, Green B. Quantification of lean bodyweight. Clin Pharmacokinet 2005;44 (10):1051-1065

20 Lemmens HJ, Bernstein DP, Brodsky JB. Estimating blood volume in obese and morbidly obese patients. Obes Surg 2006;16(06): 773-776

21 Srivastava A, Brewer AK, Mauser-Bunschoten EP, et al; Treatment Guidelines Working Group on Behalf of The World Federation Of Hemophilia. Guidelines for the management of hemophilia. Haemophilia 2013;19(01):e1-e47

22 NovoEight ${ }^{\circledR}$ Summary of Product Characteristics. Novo Nordisk A/ S, Bagsværd, Denmark. Available at: https://www.ema.europa.eu/ en/documents/product-information/novoeight-epar-productinformation_en.pdf. Accessed July 9, 2019

23 Henrard S, Hermans C. Impact of being overweight on factor VIII dosing in children with haemophilia A. Haemophilia 2016;22 (03):361-367

24 Lee SY, Ahn S, Kim YJ, et al. Comparison between dual-energy Xray absorptiometry and bioelectrical impedance analyses for accuracy in measuring whole body muscle mass and appendicular skeletal muscle mass. Nutrients 2018;10(06):pii: E738

25 Singer JP, Peterson ER, Snyder ME, et al. Body composition and mortality after adult lung transplantation in the United States. Am J Respir Crit Care Med 2014;190(09):1012-1021

26 Balistreri CR, Caruso C, Candore G. The role of adipose tissue and adipokines in obesity-related inflammatory diseases. Mediators Inflamm 2010;2010:802078

27 Fantuzzi G, Mazzone T. Adipose tissue and atherosclerosis: exploring the connection. Arterioscler Thromb Vasc Biol 2007;27 (05):996-1003 
28 Lalezari S, Martinowitz U, Windyga J, et al. Correlation between endogenous VWF:Ag and PK parameters and bleeding frequency in severe haemophilia A subjects during three-times-weekly prophylaxis with rFVIII-FS. Haemophilia 2014;20(01):e15-e22

29 Pipe SW. The hope and reality of long-acting hemophilia products. Am J Hematol 2012;87(Suppl 1):S33-S39

30 Björkman S, Folkesson A, Jönsson S. Pharmacokinetics and dose requirements of factor VIII over the age range 3-74 years: a population analysis based on 50 patients with long-term prophy- lactic treatment for haemophilia A. Eur J Clin Pharmacol 2009;65 (10):989-998

31 Björkman S, Oh M, Spotts G, et al. Population pharmacokinetics of recombinant factor VIII: the relationships of pharmacokinetics to age and body weight. Blood 2012;119(02):612-618

32 Morfini M, Marchesini E, Paladino E, Santoro C, Zanon E, Iorio A. Pharmacokinetics of plasma-derived vs. recombinant FVIII concentrates: a comparative study. Haemophilia 2015;21(02): 204-209 